\title{
EARTHQUAKE FORECASTING PROBABILITY CHARTS
}

\author{
D.A. Rhoades*
}

SUMMARY

Earthquake forecasts can be expressed in a useful form for practical purposes by mapping the probability that specific strengths of shaking will occur within specified timespans. The minimum requirements for a forecasting model to allow this form of presentation are discussed and an illustration based on the precursory swarm hypothesis is given.

\section{INTRODUCTION}

Current progress in the recognition of earthquake precursors could make possible a systematic approach to earthquake forecasting in which a long-range forecast based on the observance of a long-term precursor would be successively refined by reference to shorter term precursors occurring in the precursory area. Many different earthquake precursors have been proposed ${ }^{\text {, some }}$ of the more commonly mentioned being seismic velocity changes, fluctuations in seismicity, variations in tilt and strain and anomolous ground deformations. Short-term precursors include such phenomena as variations in the water-level of wells and unusual animal behaviour. Experience in China has shown that the observance of short-term precursors can lead to an extremely accurate prediction on which a successful evacuation can be based. However, it is doubtful whether such accuracy can be expected on a routine basis and it is notable that the Chinese experience also includes a number of false alarms and failures. In any case, effective long-range forecasting would appear to be a prerequisite for systematic short-range forecasting because the high cost of intensive monitoring for short-term precursors would preclude its being carried out over long time periods and large areas. Long-range forecasting would be of great benefit also for the purpose of taking precautionary measures aimed at mitigating the damaging effects of major earthquakes, including decisions on the fate of earthquake-risk structures and civil defence preparations.

With these purposes in mind it is appropriate to consider what is the most suitable form for a long-range forecase to take. A long-range forecast inevitably involves uncertainty in the time, magnitude and, to a lesser extent, the location of the preducted earthquakes. There is also uncertainty as to whether such earthquakes will occur at all and the possibility of unpredicted earthquakes occurring always exists. One of the main tasks facing the forecaster is to convey this uncertainty adequately. This could not usually be done merely by stating wide limits for the estimated occurrence time, magnitude and location; a statement of the variation of risk in time and space seems preferable. Furthermore,

\footnotetext{
* Applied Mathematics Division, DSIR, Wellington.
}

if precautionary measures are a major objective then the forecast is better stated in terms of intensity of shaking rather than magnitude, since the damaging effect of a given magnitude varies greatly from one place to another. Intensity probability charts, in which the probability of a given intensity of shaking occurring over a given future time interval is mapped for all locations, appear to be an ideal expression of the forecast as they bring together a rather complex set of probabilities into a simple form that is readily understood.

\section{MTNIMUM REQUIREMENTS FOR A FORECASTTING MODEL}

Before a model for forecasting based on the occurrence of precursors could be given any official status, adequate estimates of all the parameters in the model would have to be available. Although the parameters involved would depend on the forecasting hypothesis it is possible to identify several different levels of uncertainty that must be treated in all cases. The first relates to the time and magnitude of the predicted earthquake. The uncertainty here is expressed by the estimated joint distribution for the precursor time $T$ and magnitude $M$ of the predicted earthquake, given that it occurs. For many precursory phenomena a linear relationship holds between $M$ and the logarithm of $\mathrm{T}(1)$. To estimate the joint distribution it is necessary to make a direct estimate of the

distribution of either $\mathrm{T}$ or $\mathrm{M}$ from some quantifiable feature of the precursor.

A second level of uncertainty is expressed by the false alarm and failure rates estimated from past events. This stage demands that criteria by which the precursory phenomenon is recignised be stated unambiguously and a search made of past data for all occurrences of the precursor with and without following mainshocks (i.e. successes and false alarms) and for all occurrences of mainshocks without a corresponding precursor (i.e. failures). From this score-sheet of past successes, failures and false alarms the success rate and failure rate of the method can be estimated. The success rate represents the initial probability of success in future preductions given the occurrence of the precursor. This probability $\operatorname{Pr}(\mathrm{S})$ would gradually decrease as time passed without the mainshock occurring. On the other hand, confirmation of the preduction by other precursors could raise $\operatorname{Pr}(\mathrm{S})$. In either case the calculation of the adjustment is 
based on Bayes' rule. It Gt represents all information available at some time after the observance of the precursor then the conditional probability of success given $G_{t}$ is given by

$\operatorname{Pr}\left(S \mid G_{t}\right)=\frac{\operatorname{Pr}\left(G_{t} \mid S\right) \operatorname{Pr}(S)}{\operatorname{Pr}\left(G_{t} \mid S\right) \operatorname{Pr}(S)+\operatorname{Pr}\left(G_{t} \mid S\right) \operatorname{Pr}(S)}$

From the joint distribution of $T$ and $M$ and the current estimate of the probability of success the probability $\mathrm{P}_{m}\left(E_{\Delta}\right)$ of the predicted mainshock occurring in any future time-magnitude window $\Delta$ can be estimated.

$$
\operatorname{Pr}\left(S \mid G_{t}\right) \operatorname{Pr}\{(T, M) E \Delta\}
$$

$\operatorname{Pm}_{m}\left(E_{\Delta}\right)=\frac{\operatorname{Pr}(T>t)}{\operatorname{Pr}\left(S / T_{t}\right)}$

Although the above formulation is complete in that it allows estimates of all the probabilities that we shall later require, there is a further level of uncertainty which has not yet been touched on, namely the uncertainty surrounding the prediction hypothesis itself. The process by which the forecaster sets the recognition criteria for the precursory phenomenon does not lend itself to statistical analysis. The criteria would presumably be set so as to maximise the estimated success rate and to minimise the estimated failure rate. It is to be expected therefore that the estimates will be biased on the optimistic side but to what extent cannot be determined until the hypothesis has undergone a number of tests in actual prediction. While the simplicity of the criteria and the consistency of the hypothesis with other scientific theories would be factors in its favour, there is no substitute for submitting the hypothesis to future tests. The estimated success and failure rates would given an objective indication of the reliability of the hypothesis only when they were based on earthquakes occurring subsequent to the formulation of the hypothesis.

\section{INTENSITY PROBABILITY CHARTS}

For a forecasting model satisfying the conditions stated above, the expression of forecasts in terms of intensity probability charts can be given provided that formulae are available indicating the magnitude range of an earthquake at any given location to produce a given felt intensity at any other location. For New Zealand earthquakes these can be deduced from formulae given by Smith ${ }^{(2)}$. At any point the probability of a given intensity of shaking occurring due to a preducted mainshock can then be calculated under the assumption that all possible locations of the mainshock consistent with the source area lying entirely within the precursory area are equally likely. In prectice the precursory area is usually several times larger than the source area. For computational purposes the edge of the source area can be taken to fall midway between the MM IX and M X isoseismals.

The probability of a given intensity occurring at any point due to the occurrence of failures during the forecasting period can be estimated on the assumption that failures occur with the same spatial distribution as historical earthquakes but with an average return time $R_{f}$ given by

$\mathrm{R}_{\mathrm{f}}=\frac{\mathrm{R}_{\mathrm{h}}}{\theta_{\mathrm{F}}}$ where $\mathrm{R}_{\mathrm{h}}$ is the historical average return time and $\theta_{F}$ the estimated failure rate.

Although the expression of the forecast in terms of intensity probability rather than magnitude rage probabilities introduces another potential source of error into the forecast in the conversion formulae, the additional information in the resultant forecast justifies the procedure. Nevertheless, carrying out such an exercise for very high intensities ( $\mathrm{X}, \mathrm{XI}, \mathrm{XII}$ ) is not recommended as data on past occurrences of these intensities, at least in New Zealand, do not appear to be adequate.

Clearly a similar procedure could be used to estimate the probability of a given ground acceleration occurring during the forecasting period at any specified location, provided that the appropriate formulae to convert magnitudes to accelerations were available.

\section{A SAMPLE FORECAST}

The suggested method of presentation will be illustrated by a sample forecast based on the precursory swarm hypothesis proposed by Evison (3). The predictor used to estimate the joint distribution of $\mathrm{T}$ and $\mathrm{M}$ is $\mathrm{M}$, defined as the mean magnitude of the three largest earthquakes in the swarm. The details of the estimation procedures have been given elsewhere (4). The scoresheet for past events is impressive and Bayesian estimates of the success and failure rates, for mainshocks with $M>6.0$, are

$\hat{\theta}_{S}=0.86, \quad \hat{\theta}_{F}=0.25$

However the hypothesis has still to be confirmed by future tests, no successes or failures having been recorded since the formulation of the recognition criteria. The caution given above therefore applies to any forecasts using this model.

The forecast, presented in figure 1 , is based on three hypothetical precursory swarms of magnitude $M_{\mathrm{p}}=4.6$. The expected magnitude of the mainshock corresponding to such a swarm is $\mathrm{M}=6.1$ and the median precursory time about $\mathrm{T}=2.7$ years. Due to the skewness of the distribution of $T$ the risk can remain high in the precursory area long after the median time has passed. The swarms are assumed to have occurred 8 years, 5 years and 2 years prior to the forecasting date and the precursory areas are shown in figure la. The percentage during the two year period immediately following the date of the forecast is mapped in figure $\mathrm{lb}$ and the corresponding probability for intensity MM VIII in figure lc. The maps combine risks due to the occurrence of potential failures. The return times for historical earthquakes used are those given by Smith $(5)$.

The forecast reflects the trade-off that occurs between a decreasing probability of success and an increasing expected magnitude as time passes. Thus the difference between the intensity MM VII and MM VIII probabilities is less in the case of the predictions that are 5 years and 8 years old than for the two year old prediction. To a lesser extent the prediction reflects the differing responses, in terms of felt intensities, to an earthquake of a given magnitude in different regions. This effect is more graphically illustrated by the fact that a swarm of magnitude 


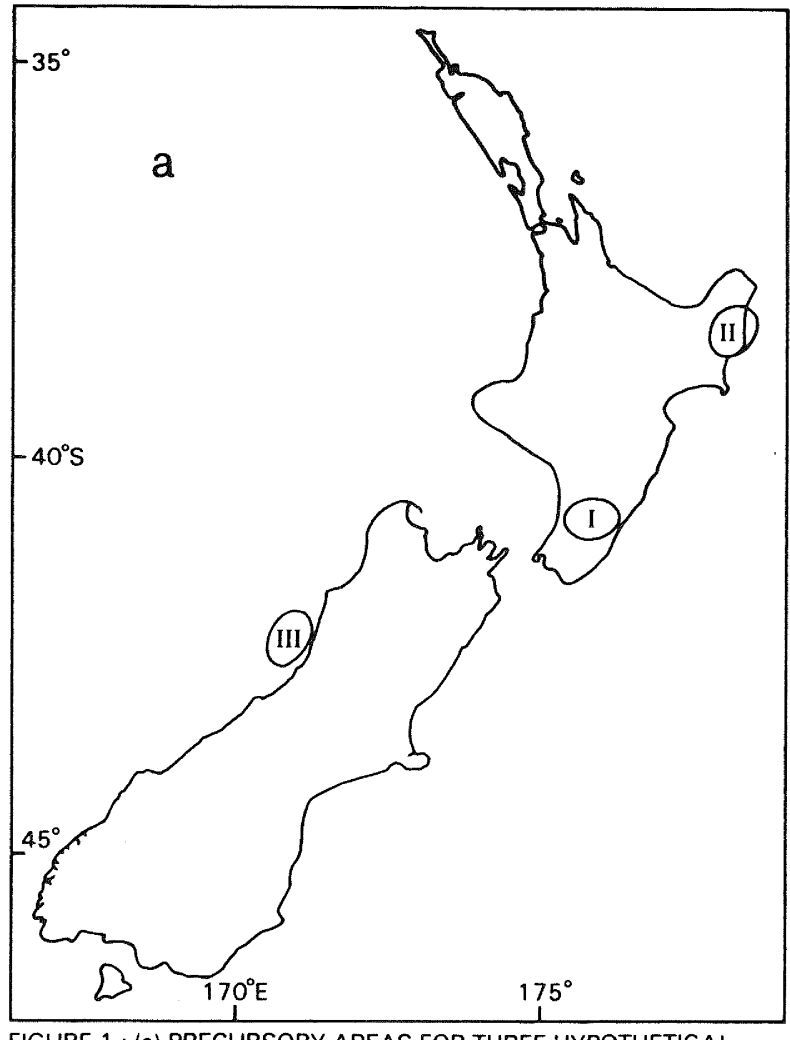

FIGURE 1 : (a) PRECURSORY AREAS FOR THREE HYPOTHETICAL

(I) 8 YEARS PRIOR TO THE FORECASTING DATE

(I) 8 YEARS PRIOR TO THE FORECASTING DATE

(II) 5 YEARS PRIOR TO THE FORECASTING DATE
(III) 2 YEARS PRIOR TO THE FORECASTING DATE

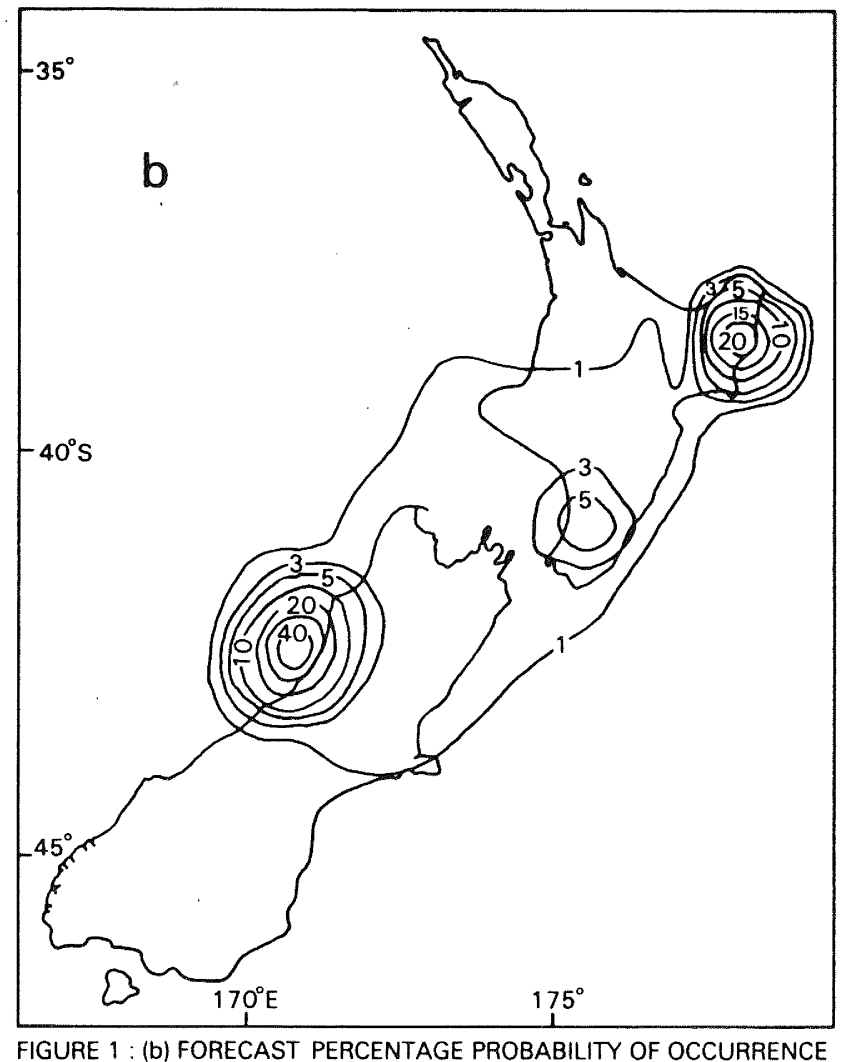

FIGURE $1 ;$ (b) FORECAST PERCENTAGE PROBABILITY OF OCCURRENCE OF INTENSTIY MM VII IN THE TWO YEAR PERIOD FOLLOWING THE TO THE PRECURSORS IN FIGURE 1 I HAVE NOT YET OCCURRED

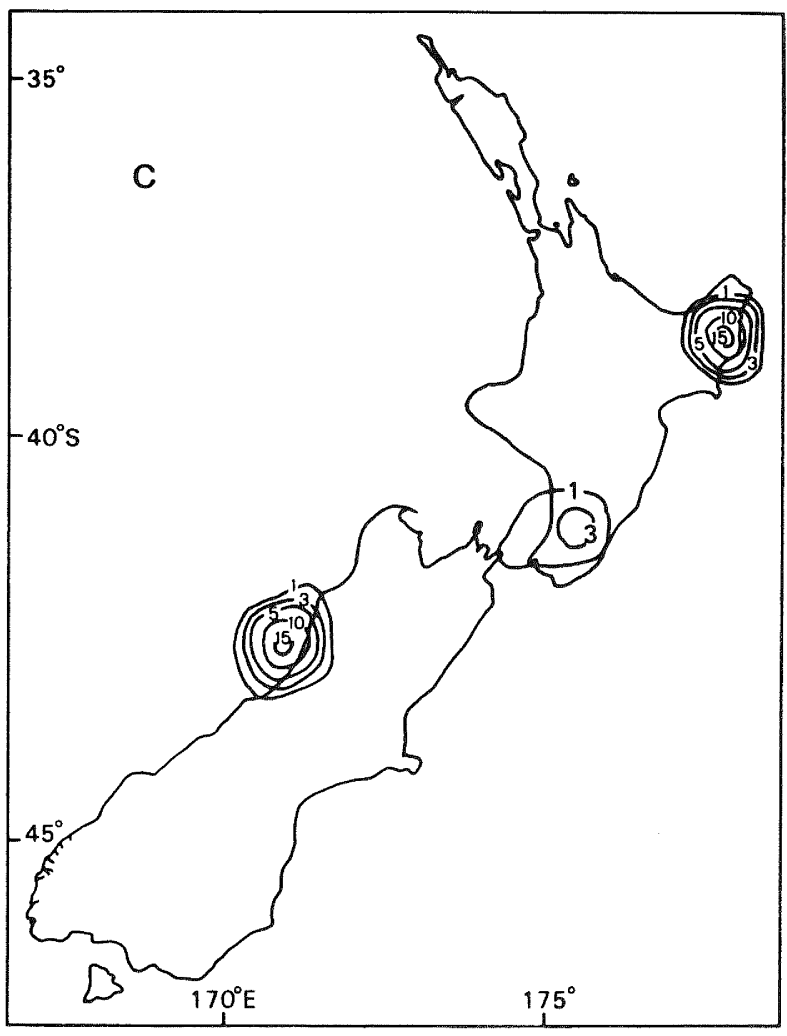

FIGURE 1 : (c) FORECAST PERCENTAGE PROBABILITY OF OCCURRENCE OF INTENSITY MM VIII IN THE TWO YEAR PERIOD CORRESPONDING TO THE PRECURSORS IN FIGURE I HAVE NOT YET OCCURRED 
4.6 occurring in the Fiordland region would not cause any change to the forecast in figures $\mathrm{lb}$ and 1c, regardless of the time of its occurrence.

\section{CONCLUSION}

Intensity probability charts are an effective means of conveying information in earthquake forecasting when the prediction involves large uncertainties. Although the example given is based on a hypothesis yet to be confirmed by a successful test, it does indicate the high degree of spatial risk concentration that can be expected from an effective long-range predictor. Intensity probability charts for several different intensities issued at periodic intervals would provide a suitable basis for committing resources to the detection of medium and short-range precursors. The observation of other precursors would surely result in even greater concentrations of risk. It is not too soon for those who would be involved in decision-making on precautionary measures to consider what would be the most apprepriate courses of action if such high levels of estimated risk were to arise.

\section{ACKNOWLEDGEMENTS}

I am grateful to Professor F. F. Evison for providing the data on the swarm hypothesis for the example given. Dr W. D. Smith kindly made available his computer sub-routines for calculating likely intensities of New Zealand earthquakes.

\section{RFFERENCES}

1. Rikitake, T., 1975, "Earthquake Precursors". Bull. Seismol. Soc. Am., 65, 2069-2074.

2. Smith, W. D., 1976, "Statistical Estimates of the Likelihood of Earthquake Shaking Throughout New Zealand". Bull. N.Z. Nat. Soc. Earthqu. Eng., 9, 213-221.

3. Evison, F. F., 1977, "The Precursory Earthquake Swarm". Phys. Earth Planet. Inter., 15, 19-23.

4. Rhoades, D. A. and Evison, F. F., 1979, "Long-Range Earthquake Forecasting Based on a Single Predictor". To be published.

5. Smith, W. D., 1978, "Spatial Distribution of Felt Intensities for N.Z. Earthquakes". N.Z. J. G. Geol. Geophys., 21, 293-31l.

This paper was presented at the South Pacific Regional Conference on Earthquake Engineering held in Wellington on 8,9 and 10 May, 1979. 